\title{
MODELOS DE CAPACIDADE DE SUPORTE DE CARGA DE UM LATOSSOLO VERMELHO-AMARELO SOB DIFERENTES USOS E MANEJOS(1)
}

\author{
Bruno Silva Pires ${ }^{(2)}$, Moacir de Souza Dias Junior ${ }^{(3)}$, Wellington Willian \\ Rocha $^{(4)}$, Cezar Francisco Araujo Junior ${ }^{(5)} \&$ Rita de Cássia Ribeiro \\ Carvalho $^{(6)}$
}

\section{RESUMO}

A compactação do solo tem sido apontada como um dos principais fatores responsáveis pela redução da produtividade de diversas culturas agrícolas. A utilização dos modelos de capacidade de suporte de carga para auxiliar na prevenção ou na identificação de manejos mais resistentes ou suscetíveis à compactação dos solos é um método testado e consolidado. Este estudo foi realizado com os seguintes objetivos: gerar modelos de capacidade de suporte de carga para os diferentes usos e manejos de um Latossolo Vermelho-Amarelo; e identificar, por meio do uso desses modelos, o uso ou manejo mais suscetível e mais resistente à compactação. O estudo foi conduzido no município de Passos, MG. Foram avaliados sete usos e manejos no solo em estudo, sendo eles: pastagem irrigada antes e após o pisoteio animal, pastagens não irrigadas antes e após o pisoteio animal, corredor de acesso aos piquetes, plantio direto de milho e mata nativa. As amostras indeformadas, coletadas na camada de 0 a $5 \mathrm{~cm}$, foram submetidas ao ensaio de compressão uniaxial. Os modelos de capacidade de

(1) Parte da Tese de Doutorado do primeiro autor apresentada ao Programa de Pós-Graduação em Ciência do Solo do Departamento de Ciência do Solo da Universidade Federal de Lavras - DCS/UFLA. Recebido para publicação em 11 de julho de 2011 e aprovado em 19 de janeiro de 2012.

(2) Engenheiro-Agrônomo, Mestre em Solos e Nutrição de Plantas. Doutorando em Ciência do Solo do Departamento de Ciência do Solo da Universidade Federal de Lavras - DCS/UFLA. Caixa Postal 3037, CEP 37200-000 Lavras (MG). Bolsista do CNPq. E-mail: piresbrunosilva@hotmail.com

(3) Engenheiro Agrícola, PhD in Crop and Soil Science, Professor Orientador Associado do Departamento de Ciência do Solo, UFLA. Bolsista CNPq. Pesquisador Mineiro da FAPEMIG. E-mail: msouzadj@dcs.ufla.br

(4) Engenheiro Agrícola, Professor Coorientador Adjunto 3 do Departamento de Agronomia da Universidade Federal dos Vales do Jequitinhonha e Mucuri, Diamantina (MG). Campus JK - Diamantina/MG, Rodovia MGT 367, km 583, nº 5000 - Alto da Jacuba. E-mail: wwillian@ufvjm.edu.br

(5) Engenheiro-Agrônomo, Mestre em Solos e Nutrição de Plantas, Doutor em Ciência do Solo. Pesquisador da Área de Solos do Instituto Agronômico do Paraná - IAPAR. Caixa Postal 48, CEP 86001-970 Londrina (PR). E-mails: cezar_araujo@iapar.br; cfaj@bol.com.br

(6) Zootecnista, Doutora em Zootecnia, Professora da Universidade do Estado de Minas Gerais, Campus de Passos. Av. Juca Stockler 1130, CEP 37900-00 Passos (MG). E-mail: ritarcarvalho@uol.com.br 
suporte de carga dos manejos pastagem irrigada e não irrigada, antes do pisoteio animal, não diferiram entre si, mas foram diferentes do modelo da mata nativa. Os modelos de capacidade de suporte de carga dos manejos pastagem irrigada após pisoteio, pastagem não irrigada após pisoteio, plantio direto de milho e corredor de acesso aos piquetes não diferiram estatisticamente, porém foram diferentes do modelo gerado para a mata nativa. Em comparação com a mata, conclui-se que o pisoteio animal alterou a estrutura do solo.

Termos de indexação: compactação do solo, pastagem, pisoteio animal.

\title{
SUMMARY: LOAD CARRYING CAPACITY MODELS OF AN OXISOL UNDER DIFFERENT USE AND MANAGEMENT
}

\begin{abstract}
Soil compaction has been identified as one of the main factors causing yield reduction of several crops. The use of the load carrying capacity models to identify management techniques which leads to alterations on soil density is a proven and consolidated methodology. This study was carried out to: a) generate load carrying capacity models for different uses and managements of an Oxisol; b) identify the land use or management that is most susceptible or most resistant to compaction by these models. The study was conducted in Passos, Minas Gerais. Seven land use and managements were evaluated in the study, namely: irrigated pastures before and after cattle trampling, non-irrigated pasture before and after cattle trampling, passage to the pickets, no-tillage corn, and native forest. Undisturbed soil samples of the 0-5 cm layer were subjected to uniaxial compression test. The load carrying capacity models of irrigated and non-irrigated pasture prior to cattle trampling did not differ from each other but from the native forest model. The load carrying capacity models of the irrigated pasture after trampling, non-irrigated pasture after trampling; no-tillage corn and passage to the pickets did not differ statistically, but did from the native forest model. Compared to the native forest, it was concluded that cattle trampling changed the soil structure.
\end{abstract}

Index terms: Soil compaction, pasture, cattle trampling.

\section{INTRODUÇÃO}

A área total de pastagens no Brasil abrange 180 milhões de hectares, o que corresponde a mais de $20 \%$ do território nacional (Zimmer et al., 2002), e um dos entraves na obtenção de elevadas produtividades tem sido a compactação do solo.

Os sistemas de manejos alteram a estrutura do solo, que por sua vez interferem nas suas propriedades físicas, químicas e biológicas, como densidade do solo, capacidade de suporte de carga do solo, formas e quantidades de nutrientes no solo, quantidade e diversidade da população macro e microbiológica (Faria et al., 1998; Kondo \& Dias Junior, 1999; Singh \& Malhi, 2006). Apesar de vários pesquisadores (Silva et al., 2000; Oliveira et al., 2003; Assis \& Lanças, 2005; Singh \& Malhi, 2006) terem identificado os efeitos do manejo nas propriedades físicas do solo, há carência de estudos que quantifiquem os níveis de pressões que podem ser aplicadas aos solos sob pastagens sem que haja degradação da estrutura.
A compreensão e a quantificação dos impactos causados pela compactação do solo têm sido uma das preocupações dos pesquisadores quando se considera a crescente mecanização associada ao aumento na capacidade operacional das máquinas e intensidade de tráfego nas áreas agrícolas (Raper, 2005; Hanza \& Anderson, 2005). Em vista disso, melhorar e preservar a qualidade da estrutura do solo em áreas sob diferentes sistemas de manejo é de fundamental importância quando se almeja maior produtividade e preservação ambiental (Secco et al., 2005; Libardi $\&$ Fernandes, 2006). A preservação da estrutura do solo está relacionada com a sua capacidade de suporte de carga, a qual pode ser obtida nos ensaios de compressão uniaxial de solos parcialmente saturados.

O entendimento do comportamento compressivo do solo adquire grande importância em razão de ser a base, do ponto de vista físico, para a escolha do manejo mais adequado visando reduzir os efeitos prejudiciais causados pelos manejos à estrutura dos solos. Levando esses aspectos em consideração, Dias Junior (1994) desenvolveu o modelo de capacidade 
de suporte de carga, que prediz a máxima pressão que o solo pode suportar para diferentes umidades, sem causar compactação adicional, em função da pressão de pré-consolidação e da umidade do solo. A pressão de pré-consolidação divide a curva de compressão do solo em duas regiões: uma de deformação pequena, elástica e recuperável (curva de compressão secundária, que é um indicativo do histórico do manejo do solo), e outra de deformação plástica e não recuperável (curva de compressão virgem), onde ocorre a compactação adicional do solo (Dias Junior \& Pierce, 1996). A pressão de pré-consolidação é a pressão que divide estas duas regiões e vem sendo utilizada por diversos pesquisadores como indicadora da capacidade de suporte de carga do solo e da qualidade estrutural (Dias Junior, 1994; Dias Junior \&Pierce, 1995; Silva et al., 2000; Oliveira, 2002; Lima, 2004; Peng et al., 2004; Dias Junior et al., 2005; Silva et al., 2006).

Em culturas irrigadas, a compactação do solo torna-se preocupante, pois este permanece a maior parte do tempo com elevada umidade e com baixa capacidade de suporte de carga, sendo, portanto, mais suscetível à compactação. Já em culturas não irrigadas a compactação pode ocorrer devido ao tráfego de máquinas em condições inadequadas de umidade ou devido ao fato de as pressões aplicadas serem maiores do que a capacidade de suporte de carga do solo (Kondo \& Dias Junior, 1999; Silva et al., 2000; Oliveira et al., 2003; Assis \& Lanças, 2005; Dias Junior et al., 2005; Silva et al., 2006), refletindo em baixa produtividade. Nas pastagens, a compactação do solo tem ocorrido devido ao pisoteio animal. Nas pastagens irrigadas a compactação ocorre com maior intensidade, por não ser possível evitar o pisoteio animal (Lima, 2004).

Ao avaliar a compressibilidade do solo sob os sistemas de pastejo rotacionado irrigado e não irrigado, Lima (2004) observou aumento na pressão de pré-consolidação no sistema de pastejo rotacionado irrigado, em relação ao sistema pastejo rotacionado não irrigado. Os animais podem exercer pressões no solo da ordem de 350 a $400 \mathrm{kPa}$ (Proffitt et al., 1993; Betteridge et al., 1999; Nie et al., 2001), mas esses autores sugerem que a pressão exercida pelos animais em movimento pode ser o dobro da exercida quando eles estão parados, pois ao movimentarem podem ficar apoiados ao solo com apenas duas patas o que torna a compactação do solo causada pelo pisoteio animal um importante assunto a ser estudado.

Com base nesses fatores, o presente estudo teve como objetivos: gerar modelos de capacidade de suporte de carga para os diferentes usos e manejos de um Latossolo Vermelho-Amarelo; e identificar, por meio desses modelos, o uso ou manejo mais suscetível e mais resistente à compactação do solo.

\section{MATERIAL E MÉTODOS}

O estudo foi conduzido na Fazenda Experimental da Universidade Estadual de Minas Gerais, no município de Passos-MG, com latitude de $19^{\circ} \mathrm{S}$ e longitude de $43^{\circ} \mathrm{W}$ de Greenwich. As áreas do estudo encontram-se a uma altitude média de $700 \mathrm{~m}$, com temperatura média anual de 18 a $20^{\circ} \mathrm{C}$ e precipitação pluvial média anual de 1.709,4 mm.

O relevo é suave ondulado em grandes extensões, com declividade entre 3 e $8 \%$. O solo da área de estudo foi classificado como Latossolo VermelhoAmarelo distrófico, com textura média (Embrapa, 2006). Nos quadros 1 e 2 são apresentadas as caracterizações físicas e químicas do solo estudado.

O estudo foi conduzido em uma área de oito hectares, dos quais dois foram de pastagem irrigada, dois de pastagem não irrigada, dois sob sistema plantio direto de milho e dois de mata nativa.

Quadro 1. Caracterização física do Latossolo Vermelho-Amarelo (LVA) localizado na Fazenda Experimental de FESP/UEMG sob diferentes manejos, na profundidade de 0-5 cm

\begin{tabular}{|c|c|c|c|c|c|c|c|c|}
\hline Manejo & Argila & Areia & Silte & MO & $\mathrm{CC}$ & PMP & Dp & Ds \\
\hline & & - $\mathrm{g} \mathrm{kg}$ & & ـ & \multicolumn{2}{|c|}{$\ldots \mathrm{kg} \mathrm{kg}^{-1} \_$} & \multicolumn{2}{|c|}{$-\mathrm{Mg} \mathrm{m}^{-3}$} \\
\hline Pia & 280 & 620 & 100 & 20 & 0,22 & 0,13 & 2,64 & 1,34 \\
\hline Pnia & 280 & 610 & 110 & 19 & 0,23 & 0,15 & 2,65 & 1,28 \\
\hline Pip & 280 & 620 & 100 & 16 & 0,26 & 0,16 & 2,64 & 1,35 \\
\hline Pnip & 270 & 620 & 110 & 20 & 0,24 & 0,15 & 2,65 & 1,38 \\
\hline Mata & 270 & 600 & 130 & 27 & 0,25 & 0,15 & 2,64 & 1,30 \\
\hline PD & 270 & 610 & 120 & 38 & 0,28 & 0,16 & 2,65 & 1,34 \\
\hline Corredor & 280 & 600 & 120 & 10 & 0,24 & 0,18 & 2,65 & 1,56 \\
\hline
\end{tabular}

Pia: pastagem irrigada antes do pisoteio animal; Pnia: pastagem não irrigada antes do pisoteio animal; Pip: pastagem irrigada após o pisoteio animal; Pnip: pastagem não irrigada após o pisoteio animal; PD: plantio direto de milho; MO: matéria orgânica; CC: capacidade de campo (amostras submetidas ao potencial mátrico de - $6 \mathrm{kPa}$ ); PMP: ponto de murcha permanente (amostras submetidas ao potencial mátrico de $-1.500 \mathrm{kPa}$ ); Dp: densidade de partículas; Ds: densidade do solo. 
Quadro 2. Caracterização mineralógica do Latossolo Vermelho-Amarelo (LVA) localizado na Fazenda Experimental da FESP/UEMG

\begin{tabular}{|c|c|c|c|c|c|c|c|}
\hline Profundidade & $\mathrm{SiO}_{2}$ & $\mathrm{Al}_{2} \mathrm{O}$ & $\mathrm{Fe}_{2} \mathrm{O}_{3}$ & $\mathrm{TiO}_{2}$ & $\mathrm{P}_{2} \mathrm{O}_{5}$ & $\mathbf{K} \mathbf{i}^{(1)}$ & $\mathbf{K r}^{(1)}$ \\
\hline $\mathrm{cm}$ & & & - \% & & - & & \\
\hline $20-40$ & 8,36 & 11,83 & 5,95 & 1,37 & 0,04 & 1,21 & 0,91 \\
\hline
\end{tabular}

(1) Ki: relação molecular $\mathrm{SiO}_{2} / \mathrm{Al}_{2} \mathrm{O}_{3} ; \mathrm{Kr}$ : relação molecular $\mathrm{SiO}_{2} /\left(\mathrm{Al}_{2} \mathrm{O}_{3}+\mathrm{Fe}_{2} \mathrm{O}_{3}\right)$.

Os quatro hectares de pastagem foram cultivados com braquiária (Brachiaria brizanta cv. Vitória), locados em faixas e divididos em 32 piquetes ao todo (16 para cada faixa), para condução de pastejo rotacionado de novilhos da raça nelore. Para implantação da forrageira, usou-se o sistema convencional, com uma aração e duas gradagens. A semeadura foi realizada com um distribuidor de calcário. As divisões dos piquetes foram feitas com cercas elétricas energizadas com eletrificadores ligados à rede elétrica e a um painel solar, e o fornecimento de água e sal mineral foi em uma área de lazer com fornecimento à vontade.

As pastagens foram submetidas a uma pressão de pastejo de cinco unidades animal por hectare, período de ocupação de dois dias e período de descanso de 32 dias. Já na área irrigada, a irrigação foi por aspersão em malha fechada. O monitoramento da irrigação foi feito utilizando-se um tanque classe A, instalado próximo ao experimento; utilizou-se um turno de rega fixo de sete dias.

Os dois hectares sob sistema de plantio direto de milho correspondem a uma área onde foi realizado o cultivo de milho sob o sistema de plantio direto há mais de sete anos. A área de mata nativa (dois hectares) situa-se abaixo da área de pastagem irrigada e serviu como referência para comparação dos atributos físicos avaliados.

As correções e as adubações de plantio e de cobertura seguiram as recomendações para o Estado de Minas Gerais (CFSEMG, 1999).

Concomitantemente com as operações de preparo do solo e implantação da pastagem, foram retiradas amostras indeformadas de solo na camada de 0 a $5 \mathrm{~cm}$, com o auxílio de um amostrador de Uhland, utilizando anéis volumétricos de $6,40 \mathrm{~cm}$ de diâmetro por 2,54 cm de altura. A amostragem constituiu-se de duas etapas: antes da entrada dos animais e seis meses após a entrada deles na área.

Antes da entrada dos animais na área, foram coletadas 64 amostras indeformadas em cada um dos sistemas de pastejo estudados; após seis meses de pastejo, foram coletadas 32 amostras indeformadas em cada sistema de pastejo.

$\mathrm{Na}$ mata nativa vizinha da pastagem e sob a mesma classe de solo, foram coletadas 32 amostras indeformadas em anéis semelhantes, e na área de plantio direto de milho foram coletadas mais 32 amostras indeformadas. Foram coletadas ainda 10 amostras indeformadas nos corredores de acesso aos piquetes, totalizando assim 266 amostras, as quais foram usadas para realizar ensaios de compressão uniaxial, além da determinação das umidades na capacidade de campo e no ponto de murcha permanente.

A parte de descarte das amostras indeformadas oriundas das partes superiores e inferiores dos anéis de amostragem foi usada para caracterização do solo, que consistiu em análise granulométrica, pelo método da pipeta (Day, 1965; Embrapa, 1997); densidade de partículas, pelo método do balão volumétrico (Blake \& Hartge, 1986b); e teor de matéria orgânica (Raij \& Quaggio, 1983) (Quadro 1). Foram determinados os teores de $\mathrm{Si}, \mathrm{Al}, \mathrm{Fe}, \mathrm{P}$ e Ti, por meio do ataque por $\mathrm{H}_{2} \mathrm{SO}_{4}$ (Embrapa, 1997). Os índices $\mathrm{Ki}$ e $\mathrm{Kr}$ foram calculados, respectivamente, pelas relações moleculares $\mathrm{SiO}_{2} / \mathrm{Al}_{2} \mathrm{O}_{3}$ e $\mathrm{SiO}_{2} /\left(\mathrm{Al}_{2} \mathrm{O}_{3}\right.$ $+\mathrm{Fe}_{2} \mathrm{O}_{3}$ ) (Quadro 2).

Para a determinação das umidades na capacidade de campo e ponto de murcha permanente do solo, foram utilizadas as amostras indeformadas, sendo estas amostras saturadas e equilibradas às tensões de -6 e $-1.500 \mathrm{kPa}$, respectivamente, no extrator de Richards (Embrapa, 1997). Após atingirem o equilíbrio, essas amostras foram pesadas, e as umidades, determinadas pelo método termogravimétrico (Quadro 1).

Para obtenção dos modelos de capacidade de suporte de carga (CSC), amostras indeformadas com diferentes umidades foram submetidas ao ensaio de compressão uniaxial de acordo com Bowles (1986) modificado por Dias Junior (1994). Para obtenção das diferentes umidades, as amostras indeformadas foram inicialmente saturadas e, a seguir, secas ao ar no laboratório. A faixa de umidade trabalhada compreendeu o intervalo entre 3 e $43 \mathrm{~kg} \mathrm{~kg}^{-1}$, sendo as amostras distribuídas de maneira homogênea dentro dessa faixa, em função da quantidade de amostras utilizadas para obtenção dos modelos. Após obtenção da umidade desejada, as amostras foram submetidas ao ensaio de compressão uniaxial, utilizando um consolidômetro da marca Boart Longyear. 
As pressões aplicadas a cada amostra obedeceram à seguinte ordem: 25, 50, 100,200,400, $800 \mathrm{e}$ $1.600 \mathrm{kPa}$. Cada pressão foi aplicada até que $90 \%$ de deformação máxima fosse alcançada (Taylor, 1948); após essa condição ser alcançada, foi aplicada uma nova pressão. Após a liberação da pressão, as amostras foram secas em estufa a $105-110^{\circ} \mathrm{C}$, por $48 \mathrm{~h}$; a densidade do solo foi determinada de acordo com Blake \& Hartge (1986a). A pressão de pré-consolidação foi obtida para cada umidade gravimétrica, de acordo com Dias Junior \& Pierce (1995), a partir da curva de compressão do solo (Figura 1).

As pressões de pré-consolidação obtidas no ensaio de compressão uniaxial foram representadas no eixo y, e as umidades, no eixo x. Por meio do software Sigma Plot 8.0 (2002), as equações matemáticas que representam os modelos de capacidade de suporte de carga do solo foram ajustadas utilizando-se o modelo proposto por Dias Junior (1994), expresso pela equação $\sigma_{p}=10^{(\mathrm{a}+\mathrm{bU})}$, em que $\sigma_{\mathrm{p}}$ é a pressão de pré-consolidação; $U$, a umidade gravimétrica do solo; e "a" e "b", os coeficientes de ajuste da regressão. As comparações das regressões foram feitas empregando-se o procedimento descrito em Snedecor \& Cochran (1989).

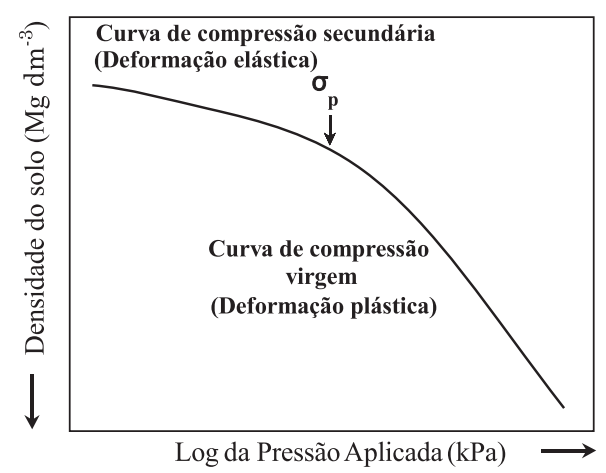

Figura 1. Curva de compressão do solo. Adaptado Dias Junior (1994).

\section{RESULTADOS E DISCUSSÃO}

Os modelos de capacidade de suporte de carga para os diferentes manejos encontram-se na figura 2. No quadro 3 encontra-se o resumo da análise estatística (comparações dos modelos de capacidade de carga $\left.\sigma_{\mathrm{p}}=10^{(\mathrm{a}+\mathrm{bU})}\right)$.

Os modelos de CSC do LVA para a pastagem irrigada antes do pisoteio animal (Pia) e pastagem não irrigada antes do pisoteio animal (Pnia) não se diferenciaram estatisticamente (Quadro 3), sendo, portanto, uma única equação ajustada a todos os valores de $\sigma_{\mathrm{p}}$ e U (Figura 3), obtendo-se assim um único modelo de CSC para esses manejos $\left(\sigma_{\mathrm{p}}=10^{(2,73-2,73 \mathrm{U})}\right.$ $\left.\mathrm{R}^{2}=0,80^{* *}\right)$.

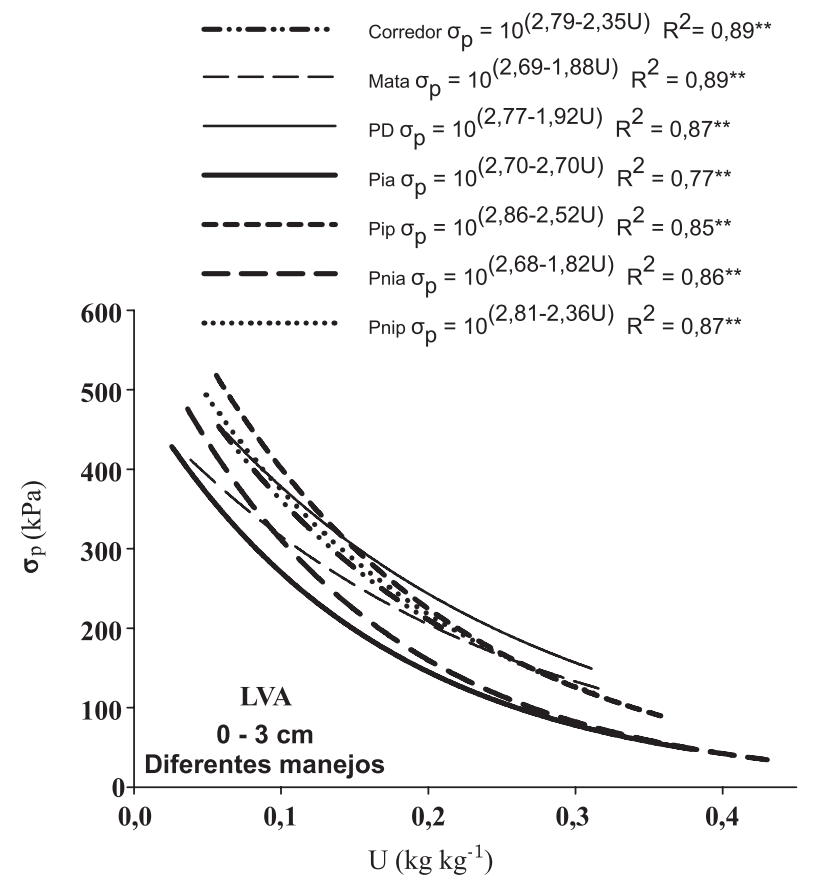

Figura 2. Modelos de capacidade de suporte de carga dos diferentes usos e manejos.

Comparando esse modelo de CSC com o modelo de CSC da área sob mata nativa, observa-se que eles foram estatisticamente diferentes, sendo a CSC da mata superior à das pastagens antes do pisoteio animal (Figura 3). Esse fato pode ser explicado pelo método de preparo de solo das pastagens, que destruiu a estrutura dessas áreas, deixando-as com menor CSC.

Os modelos de CSC do LVA para a pastagem irrigada após o pisoteio animal (Pip) e pastagem não irrigada após o pisoteio animal (Pnip) não diferenciaram estatisticamente entre si (Quadro 3), sendo, portanto, uma única equação ajustada a todos os valores de $\sigma_{\mathrm{p}}$ e $\mathrm{U}$, obtendo-se assim um único modelo de CSC para esses manejos. Esse resultado pode ser explicado pela elevada precipitação ocorrida no período de pisoteio animal, fazendo com que a área de pastagem não irrigada permanecesse durante um grande período com elevada umidade. Outro fato que pode explicar a não diferença estatística dos modelos de CSC é o curto período de tempo em que esse solo foi submetido ao pisoteio animal (seis meses). Resultados semelhantes foram encontrados por Lima (2004) quando o tempo de pisoteio foi de 108 dias.

Os modelos de CSC do LVA para a pastagem irrigada após o pisoteio animal (Pip) e pastagem não irrigada após o pisoteio animal (Pnip), quando comparadas com o plantio direto de milho, não diferenciaram estatisticamente (Quadro 3), sendo, portanto, uma única equação ajustada a todos os valores de $\sigma_{p}$ e $\mathrm{U}$, obtendo-se assim um único 
Quadro 3. Teste de significância de acordo com Snedecor \& Cochran (1989) entre os modelos de capacidade de suporte de carga $\left[\sigma_{p}=10^{(a+b U)}\right]$ de um Latossolo Vermelho-Amarelo, na camada de 0-5 cm, para os diferentes usos e manejos

\begin{tabular}{|c|c|c|c|}
\hline \multirow[b]{2}{*}{ Manejo } & \multirow[b]{2}{*}{$\mathbf{F}$} & \multicolumn{2}{|c|}{$\mathbf{F}$} \\
\hline & & $\begin{array}{l}\text { Coeficiente } \\
\text { angular, b }\end{array}$ & $\begin{array}{l}\text { Coeficiente } \\
\text { linear, a }\end{array}$ \\
\hline $\begin{array}{l}\text { Pastagem irrigada antes do pisoteio animal (Pia) vs pastagem não irrigada } \\
\text { antes do pisoteio animal (Pnia) }\end{array}$ & $\mathrm{H}$ & $\mathrm{ns}$ & $\mathrm{ns}$ \\
\hline $\begin{array}{l}\text { Pastagem irrigada antes do pisoteio animal (Pia) e pastagem não irrigada } \\
\text { antes do pisoteio animal (Pnia) vs mata nativa (MN) }\end{array}$ & $\mathrm{NH}$ & $\mathrm{ns}$ & $* *$ \\
\hline $\begin{array}{l}\text { Pastagem irrigada após o pisoteio animal (Pip) vs pastagem não irrigada } \\
\text { após o pisoteio animal (Pnip) }\end{array}$ & $\mathrm{H}$ & $\mathrm{ns}$ & $\mathrm{ns}$ \\
\hline $\begin{array}{l}\text { Pastagem irrigada após o pisoteio animal (Pip) e pastagem não irrigada } \\
\text { após o pisoteio animal (Pnip) vs plantio direto (PD) }\end{array}$ & $\mathrm{H}$ & $\mathrm{Ns}$ & $\mathrm{Ns}$ \\
\hline $\begin{array}{l}\text { Pastagem irrigada após o pisoteio animal (Pip), pastagem não irrigada após } \\
\text { o pisoteio animal (Pnip) e plantio direto (PD) vs corredor }\end{array}$ & $\mathrm{H}$ & $\mathrm{Ns}$ & $\mathrm{Ns}$ \\
\hline $\begin{array}{l}\text { Pastagem irrigada após o pisoteio animal (Pip), pastagem não irrigada após } \\
\text { o pisoteio animal (Pnip), plantio direto (PD) e corredor vs mata nativa (MN) }\end{array}$ & $\mathrm{H}$ & $\mathrm{Ns}$ & $* *$ \\
\hline $\begin{array}{l}\text { Pastagem irrigada após o pisoteio animal (Pip), pastagem não irrigada } \\
\text { após o pisoteio animal (Pnip), plantio direto (PD) e corredor vs pastagem } \\
\text { irrigada antes do pisoteio animal (Pia) e pastagem não irrigada antes do } \\
\text { pisoteio animal (Pnia). }\end{array}$ & $\mathrm{NH}$ & $\mathrm{Ns}$ & $* *$ \\
\hline
\end{tabular}

F: testa a homogeneidade dos dados; b: coeficiente angular da regressão linearizada; a: intercepto da regressão linearizada; H: homogêneo; NH: não homogêneo; ns: não significativo; ${ }^{*} \mathrm{e} * *$ significativo a 1 e $5 \%$, respectivamente.

modelo de CSC para esses manejos. A não diferença estatística dos modelos de CSC nesses manejos pode ser explicada pelo não revolvimento periódico do solo, o que preserva uma camada superficial com maior CSC devido ao pisoteio animal (Kondo, 1998) ou tráfego de máquinas (Raper, 2005; Hanza \& Anderson, 2005), ou pela consolidação da camada superficial, como no plantio direto (Assis \& Lanças, 2005).

Os modelos de CSC do LVA para a pastagem irrigada após o pisoteio animal (Pip), pastagem não irrigada após o pisoteio animal (Pnip) e plantio direto de milho, quando comparados com o modelo de CSC do corredor de acesso aos piquetes, não diferenciaram estatisticamente (quadro 3), sendo, portanto, uma única equação ajustada a todos os valores de $\sigma_{\mathrm{p}}$ e U, obtendo-se dessa forma um único modelo de CSC para esses manejos $\left(\sigma_{\mathrm{p}}=10^{(2,81-2,27 \mathrm{U})}\right.$ $\mathrm{R}^{2}=0,85^{* *}$ ) (Figura 3 ). Os corredores de acesso aos piquetes sofrem maior e mais frequente pisoteio animal, o que poderia promover maior CSC do solo. No entanto, esse comportamento não foi observado neste estudo em razão do pouco tempo de pisoteio e da chuva elevada.

Comparando esse modelo de CSC com o modelo de CSC da mata nativa, observa-se que eles foram estatisticamente diferentes, sendo a CSC da mata inferior à das pastagens após o pisoteio animal (Pnip e Pip), plantio direto e corredor (Figura 3).

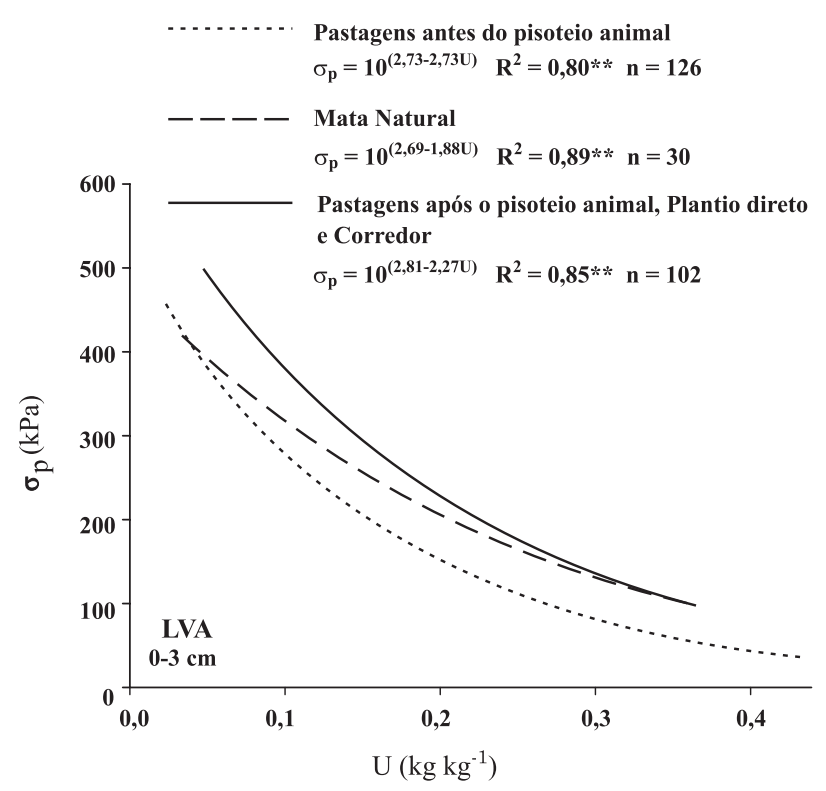

Figura 3. Modelos de capacidade de suporte de carga dos diferentes usos e manejos. 
Como a mata nativa é um referencial de qualidade estrutural, os manejos pastagem irrigada após o pisoteio animal (Pip), pastagem não irrigada após o pisoteio animal (Pnip), plantio direto de milho e corredor de acesso aos piquetes aumentaram a CSC do LVA estudado, sendo, portanto, os manejos que degradam a estrutura do solo.

O modelo de CSC das pastagens após o pisoteio animal (Pip e Pnip) apresentou maior capacidade de suporte de carga, quando comparado com o modelo de CSC das pastagens antes do pisoteio animal (Pia e Pnia), em toda a faixa de umidade estudada (Figura 3), o que comprova o efeito compactante do pisoteio animal na camada superficial do solo, podendo resultar em alteração da estrutura, selamento superficial e consequente aumento da enxurrada e da erosão, pela baixa taxa de infiltração resultante dessa compactação.

\section{CONCLUSÕES}

1. Os manejos pastagem irrigada e não irrigada, antes do pisoteio animal, geraram modelos de capacidade de suporte de cargas semelhantes, expressa pela equação $\sigma_{\mathrm{p}}=10^{(2,73-2,73 \mathrm{U})}$. Esses manejos não degradam a estrutura do solo.

2. Os manejos pastagem irrigada após pisoteio, pastagem não irrigada após pisoteio, plantio direto de milho e corredor de acesso aos piquetes geraram modelos de capacidade de suporte de carga semelhantes, expressa pela equação $\sigma_{\mathrm{p}}=10^{(2,81-2,27 \mathrm{U})}$. Esses manejos degradam a estrutura do solo.

3. O pisoteio animal após a colheita de milho em áreas sob plantio direto não causou compactação adicional.

\section{AGRADECIMENTOS}

Ao CNPq pela concessão da bolsa de estudos. À FAPEMIG pelo financiamento para instalação do experimento. À FESP/UEMG pela disponibilização da área. À UFLA pelo curso de doutorado e disponibilização dos laboratórios.

\section{LITERATURA CITADA}

ASSIS, R.L. \& LANÇAS, K.P. Avaliação da compressibilidade de um Nitossolo Vermelho distroférrico sob sistema plantio direto preparo convencional e mata nativa. R. Bras. Ci. Solo, 29:507-514, 2005.
BLAKE, G.R. \& HARTGE, K.H. Bulk density. In: KLUTE, A., ed. Methods of soil analysis. 2.ed. Madison, ASA/SSSA. 1986a. Part 1. p.363-375.

BLAKE, G.R. \& HARTGE, K. H. Partycle density, In: KLUTE, A., ed. Methods of soil analysis. 2.ed. Madison, ASA, 1986b. Part 1. p.377-382. (Agron. Monogr. 9)

BETTERIDGE, K.; MACKAY, A.D.; SHEPHERD, T.G.; BARKER, D.J.; BUDDING, P.J.; DEVANTIER, B.P. \& COSTALL, D.A. Effect of cattle and sheep treading on surface configuration of a sedimentary hill soil. Austr. J. Soil Res., 37:743-760, 1999.

BOWLES, J.E. Engineering properties of soils and their measurements. 3.ed. New York, McGraw-Hill, 1986. 218p.

COMISSÃO DE FERTILIDADE DO SOLO DO ESTADO DE MINAS GERAIS - CFSEMG. Recomendação para o uso de corretivos e fertilizantes em Minas Gerais. ( $5^{\text {a }}$ Aproximação). Viçosa, MG, 1999. 359p.

DAY, P.R. Particle fractionation and particle-size analysis. In: BLACK, C.A., ed. Methods of soil analysis. Madison, America Society of Agronomy, 1965. Part.I. p.545-567.

DIAS JUNIOR, M.S. Compression of three soils under longterm tillage and wheel traffic. East Lansing, Michigan State University, 1994. 114p. (Tese de Doutorado)

DIAS JUNIOR, M.S.; LEITE, F.P.; LASMAR JUNIOR, E. \& ARAUJO JUNIOR, C.F. Traffic effects on the soil preconsolidation pressure due to Eucalyptus harvest operations. Sci.Agric., 62:248-255, 2005.

DIAS JUNIOR, M.S. \& PIERCE, J.F. Revisão de literatura. O processo de compactação do solo e sua modelagem. R. Bras. Ci. Solo, 20:175-182, 1996.

DIAS JUNIOR, M.S. \& PIERCE, F.J. A simple procedure for estimating preconsolidation pressure from soil compression curves. Soil Technol., 8:139-151, 1995.

EMPRESA BRASILEIRA DE PESQUISA AGROPECUÁRIA EMBRAPA. Centro Nacional de Pesquisas do Solo. Manual de métodos de análise de solo. 2.ed. Brasília, Produção de informação, 1997. 212p.

EMPRESA BRASILEIRA DE PESQUISA AGROPECUÁRIA EMBRAPA. Centro Nacional de Pesquisas do Solo. Sistema brasileiro de classificação de solos. 2.ed. Rio de Janeiro, Embrapa Solos, 2006. 306p.

FARIA, J.C.; SCHAEFER, C.E.R.; RUIZ, H.A. \& COSTA, L.M. Effects of weed control on physical and micropedological properties of brazilian Ultisol. R. Bras. Ci. Solo, 22:731$741,1998$.

HANZA, M.A. \& ANDERSON, W.K. Soil compaction in copping systems a review of the nature, causes and possible solutions. Soil Till. Res., 82:121-145, 2005.

KONDO, M.K. Compressibilidade de três Latossolos sob diferentes usos. Lavras, Universidade Federal de Lavras, 1998. 121p. (Tese de Mestrado) 
KONDO, M.K. \& DIAS JUNIOR, M.S. Compressibilidade de três Latossolos em função da umidade e uso. R. Bras. Ci. Solo, 23:211-218, 1999.

LIBARDI, P.L. \& FERNANDES, F.C.S. Avaliação da qualidade do solo a partir de indicadores físico-hídricos do solo. In: REUNIÃO BRASILEIRA DE MANEJO E CONSERVAÇÃO DO SOLO E DA ÁGUA, 16., Aracaju, 2006. Palestras... Aracaju, Sociedade Brasileira de Ciência do Solo, 2006.

LIMA, C.L.R. Compressibilidade de solos versus intensidade de tráfego em um pomar de laranja e pisoteio animal em pastagem irrigada. Piracicaba, Escola Superior de Agricultura Luiz de Queiroz, 2004. 70p. (Tese de Doutorado)

NIE, Z.N.; WARD, G.N. \& MICHAEL, A.T. Impact of pugging by dairy cows on pastures and indicators of pugging damage to pasture soil on south - Western Victoria. Austr. J. Agric. Soil Res., 52:37-43, 2001.

OLIVEIRA, G.C. Alterações estruturais e comportamento compressivo de um Latossolo submetido a diferentes sistemas de manejo por 20 anos no cerrado. Lavras, Universidade Federal de Lavras, 2002. 78p. (Tese de Doutorado)

OLIVEIRA, G.C.; DIAS JUNIOR, M.S.; RESCK, D.V.S. \& CURI, $\mathrm{N}$. Alterações estruturais e comportamento compressivo de um Latossolo Vermelho distrófico argiloso sob diferentes sistemas de uso e manejo. Pesq. Agropec. Bras., 38:291$299,2003$.

PENG, S.H.; HORN, R.; ZHANG, B. \& ZAHO, Q.G. Mechanisms of soil vulnerability to compaction of homogenized and recompacted Ultisols. Soil Till. Res., 76:125-137, 2004.

PROFFITT, A.P.B.; BENDOTTI, S.; HOWELL, M.R. \& EASTHAM, J. The effect of sheep trampling and grazing on soil physical properties and pasture growth for a Red -Brown earth. Austr. J. Agric. Soil Res., 44:317-331, 1993.

RAJI, B.van \& QUAGGIO, J.A. Métodos de análise de solo para fins de fertilidade. Campinas, Instituto Agronômico de Campinas, 1983. 21p. (Boletim Técnico, 81)
RAPER, D.L. Agricultural traffic impacts on soil. J. Terramechanics, 42:259-280, 2005.

SECCO, D.; REINERT, D.J.; REICHERT, J.M. \& FERREIRA, F.P. Parâmetros de compressibilidade de um Latossolo influenciados pelo estado inicial de compactação e grau de saturação em água. In: CONGRESSO BRASILEIRO DE CIÊNCIA DO SOLO, 30., Recife, 2005. Palestra... Recife, Sociedade Brasileira de Ciência do Solo, 2005.

SIGMA PLOT. Scientific Graphing Software. Versão 8.0. San Rafael, Jandel Corporation, 2002.

SILVA, A.R.; DIAS JUNIOR, M.S.; GUIMARÃES, P.T.G. \& ARAUJO JUNIOR, C.F. Modelagem da capacidade de suporte de carga e quantificação dos efeitos das operações mecanizadas em um Latossolo Amarelo cultivado com cafeeiros. R. Bras. Ci. Solo, 30:207-216, 2006.

SILVA, V.R.; REINERT, D.J. \& REICHERT, J.M. Suscetibilidade à compactação de um Latossolo Vermelho-Escuro e de um Podzólico Vermelho-Amarelo. R. Bras. Ci. Solo, 24:239$249,2000$.

SINGH, B. \& MALHI, S.S. Response of soil physical properties to tillage and residue management on two soils in a cool temperate environment. Soil Till. Res., 85:143-153, 2006.

SNEDECOR, G.W. \& COCHRAN, W.G. Statical methods. 8.ed. Ames, Iowa State University Press, 1989.

TAYLOR, D.W. Fundamentals of soil mechanics. New York, John Wiley, 1948. 770p.

ZIMMER, A.; SILVA, M.P. \& MAURO, R. Sustentabilidade e impactos ambientais da produção animal em pastagem. In: PEIXOTO, A.M.; MAURA, J.C.; PEDREIRA, C.G.S. \& FARIA, V.P, ed. Inovações tecnológicas no manejo de pastagens. Piracicaba, FEALQ, 2002. p.31-58. 